\title{
Immunolocalization of Sprouty-1 and Sprouty-2 in Developing Rat Lung
}

\author{
Shuichi Hashimoto Hiroshi Nakano Yuko Suguta Gurmukh Singh \\ Sikandar L. Katyal \\ Department of Pathology, University of Pittsburgh School of Medicine, Pittsburgh, Pa., USA
}

\section{Key Words}

Lung $\cdot$ Development $\cdot$ Immunolocalization $\cdot$ Sprouty-1 •

Sprouty-2 $\cdot$ Fibroblast growth factor- $10 \cdot$ Caveolin

\begin{abstract}
Objective: Sprouty, a common antagonist of fibroblast growth factor (FGF) and epidermal growth factor signaling, is a key player regulating tracheal branching and eye development in Drosophila. Four Sprouty homologs have been identified in vertebrates and all share a cysteine-rich region. However, the physiological function(s) of the individual Sprouty homologs is unknown. mRNA of Sprouty homologs is expressed during mouse lung development. In the present study, we investigated the immunolocalization of Sprouty proteins in rat lung at different stages of development. Methods: Rabbit antibodies were raised against peptides derived from rat Sprouty- 1 and Sprouty-2 and were used in Western blot analysis to determine Sprouty distribution in subcellular fractions (pellets and supernatant centrifuged at 5,000 and 20,000 $\mathrm{g}$ ) and bronchoalveolar lavage fluid (BAL) from adult rat lungs or used in immunohistochemistry. $\boldsymbol{R e}$ sults: Western blot analysis revealed a 30-kDa Sprouty-1 band and a 34-kDa Sprouty-2 band in the supernatant and pellet fractions centrifuged at 20,000 g. BAL contained a
\end{abstract}

band of approximately $16 \mathrm{kDa}$ with Sprouty-1 antibody derived from proteolytic fragmentation of Sprouty-1. In embryonic day (E) 14 and E16 lungs, Sprouty-1 and Sprouty-2 were expressed both in epithelial and peripheral mesenchymal cells. In adult rat lung, bronchiolar and alveolar type II epithelial cells showed staining for both Sprouty-1 and Sprouty-2. Sprouty-1 expression was also seen in alveolar type I epithelial cells. Conclusion: In light of the proximity of the distribution of Sprouty to that of FGF-10 (peripheral mesenchyme) and its receptor FGFR2IIIb (distal tubular epithelium) in lung development, and the finding that FGF-9, which is expressed in mesothelial cells, upregulates FGF-10, it appears that Sprouty expression in epithelial and mesenchymal cells during branching morphogenesis is closely related to signaling by FGF-9 and FGF-10.

Copyright $\odot 2012$ S. Karger AG, Basel

\section{Introduction}

Lung development begins with outpouching of the foregut endoderm into surrounding mesoderm. The initial bud undergoes repetitive branching (branching morphogenesis), which is regulated by mesenchymal-endodermal interactions through the release of a variety of

\section{KARGER}

(c) 2012 S. Karger AG, Basel

Fax +41613061234

E-Mail karger@karger.ch

www.karger.com
Shuichi Hashimoto

Department of Pathology and Experimental Medicine

Kumamoto University Graduate School of Medical Sciences

Basic Medical Research Building 3F, 1-1-1 Honjo, Kumamoto 860-8556 (Japan)

Tel. +81 96373 5088, E-Mail sh6749@ my.email.ne.jp 
growth factors and other signaling molecules $[1,2]$. Of the latter, Sprouty acts as a common antagonist of a number of growth factors and participates in organogenesis [3-7]. Sprouty was first discovered during genetic studies on Drosophila [4] and shown to regulate tracheal branching and eye development in Drosophila [3-5, 7]. At least 4 Sprouty homologs (Sprouty-1, $-2,-3$ and -4 ) have been identified in vertebrates, and all share a C-terminal cysteine-rich domain $[8,9]$. The question of whether Sprouty, similar to its function in Drosophila tracheal branching, also regulates lung branching in vertebrates has been addressed [6, 10]. Inhibition of Sprouty-2 expression with antisense oligonucleotides in cultured mouse lung explants enhanced branching $[6,10]$, indicating that Sprouty- 2 acts as an inhibitor of lung branching morphogenesis.

Several studies have demonstrated that Sprouty-1 and Sprouty-2 mRNAs are expressed at various stages of lung development and in adults [6,9-12]. This report compares the distribution of Sprouty- 1 and Sprouty-2 proteins by immunohistochemistry in developing and adult rat lung. Our results indicate that Sprouty-1 and Sprouty-2 have both distinct and overlapping patterns of expression in developing lung, beginning with the onset of branching through to the adult stage. This report is the first to show that Sprouty-1 and -2 proteins are expressed by adult alveolar epithelial type II cells. Furthermore, Western blot analysis of bronchoalveolar lavage fluid (BAL) from adult rat lungs using Sprouty-1 antibody revealed the presence of a $16-\mathrm{kDa}$ polypeptide in BAL, possibly released after proteolysis of the nascent protein.

\section{Materials and Methods}

Animals

Timed pregnant rats of the Sprague-Dawley strain were obtained from Hilltop (Scottsdale, Pa., USA) and housed in pathogen-free conditions in accordance with institutional guidelines.

\section{PCR Cloning of Rat Sprouty Homologs}

Rat Sprouty-1 and Sprouty-2 cDNAs were obtained by RT-PCR using RNA obtained from adult rat lungs and PCR primers derived from published mouse cDNA sequences [9]. The products were amplified using AmpliTaq DNA polymerase (Perkin Elmer Cetus). After initial denaturation at $95^{\circ} \mathrm{C}$ for $5 \mathrm{~min}$, the samples were amplified for 40 cycles, each consisting of annealing at $55^{\circ} \mathrm{C}$ for $1 \mathrm{~min}$, elongation at $72^{\circ} \mathrm{C}$ for $3 \mathrm{~min}$ and denaturation at $95^{\circ} \mathrm{C}$ for $40 \mathrm{~s}$. The final elongation was for $15 \mathrm{~min}$ at $72^{\circ} \mathrm{C}$. The amplified DNA was cloned into the TA vector (Invitrogen) and sequenced on an ABI Prism 377 sequencer at the University of Pittsburgh, School of Medicine Research Support Facilities.
Antibodies

Rat Sprouty-1 and Sprouty-2 amino acid sequences were deduced from the nucleotide sequences and used to select peptides for raising polyclonal antibodies. Sprouty-1 and Sprouty- 2 antibodies were prepared by Bethyl Laboratories (Montgomery, Tex., USA) by immunizing rabbits with the KLH-conjugated peptides (rat Sprouty-1 peptide: AVEGRQRLDYDRDT; rat Sprouty-2 peptide: QPPRLQPSQVHSSRA). The peptides for immunization were selected from the more divergent $\mathrm{N}$-terminal amino acid sequences of Sprouty-1 and Sprouty-2 (the homology at the N-terminal sequence was $43 \%$, compared with $68 \%$ at the cysteine-rich C-terminal sequence). The antibodies were affinity purified on bound-peptide columns prior to use in Western blot analysis and immunocytochemistry. A caveolin-1 antibody was obtained from Dako (Carpinteria, Calif., USA).

\section{Fractionation of Rat Lung Homogenate}

Adult rat lungs were homogenized in $0.25 \mathrm{M}$ sucrose containing protease inhibitor cocktail (Roche, Indianapolis, Ind., USA) and centrifuged at $6,500 \mathrm{rpm}(5,000 \mathrm{~g})$ in a Beckman JA20 rotor/ J2-21 centrifuge (Beckman Instruments, Palo Alto, Calif., USA) to obtain a pellet $(6.5 \mathrm{~K}$ pellet). The supernatant was then centrifuged at $13,000 \mathrm{rpm}(20,000 \mathrm{~g})$ to obtain supernatant (13 K supernatant) and pellet ( $13 \mathrm{~K}$ pellet) fractions. BAL was collected by intratracheal instillation of normal saline into lungs of adult rats that were given lethal doses of Nembutal. Cell debris was removed from BAL by centrifugation, and the clear supernatant was concentrated by ultrafiltration.

\section{Western Blot}

The 6.5 and $13 \mathrm{~K}$ pellets, $13 \mathrm{~K}$ supernatant and BAL were subjected to Western blot analysis using Sprouty-1 or Sprouty-2 antibody. Samples were electrophoresed on 10\% acrylamide gels (Novex, San Diego, Calif., USA), and Western blot analysis was carried out using a commercial kit (Western Breeze, Novex).

Type II Cell Isolation and Northern Blot

Alveolar epithelial type II cells were isolated from adult rats, and Northern blot analysis was performed according to our previously described protocols [13-15]. Briefly, total RNA extracted from adult rat lung and isolated type II cells were fractionated in $1 \%$ agarose gels containing $0.66 \mathrm{M}$ formaldehyde. Gels with ethidium bromide-stained bands were photographed under UV light and treated with $0.05 \mathrm{~N} \mathrm{NaOH}$ prior to transfer to GeneScreen Plus (NEN Research Products, Boston, Mass., USA) with a Transvac Blotting System (Hoefer Scientific, San Francisco, Calif., USA). Blots were exposed to UV light in a UV Stratalinker (Stratagene, San Diego, Calif., USA). Northern blot was sequentially hybridized with ${ }^{32} \mathrm{P}$-labeled cDNA probes for Sprouty-1 and Sprouty-2.

Immunohistochemisry

Lung tissue was fixed in $4 \%$ paraformaldehyde, embedded in OCT compound and frozen at $-80^{\circ} \mathrm{C}$. Frozen sections were stained with antibody using the EnVision System (Dako) and counterstained with hematoxylin.

In situ Hybridization

The procedure using non-RI probes is detailed in our earlier publication [11]. Alkaline phosphatase and peroxidase substrates were Fast Red and diaminobenzidine, respectively. 
Fig. 1. Characterization of Sprouty-1 antibody by Western blot analysis of lung subcellular fractions. Three fractions $(6.5 \mathrm{~K}$ pellet, $13 \mathrm{~K}$ pellet, membrane-rich and $13 \mathrm{~K}$ supernatant) were obtained by differential centrifugation of homogenates of adult rat lungs. An additional fraction was obtained by concentrating BAL obtained from adult rat lungs. A Each fraction obtained by differential centrifugation shows the presence of caveolin-1. The highest amount of caveolin was seen in the $13 \mathrm{~K}$ pellet fraction. These fractions were further used for characterization of Sprouty-1 polyclonal antibody by Western blot analysis. B Blot treated with Sprouty-1 antibody. C Blot treated with peptide-absorbed Sprouty-1 antibody. Specific bands of approximately 16 and 30 $\mathrm{kDa}$ were detected in the BAL and $13 \mathrm{~K}$ supernatant fractions, respectively (B). These bands were not detectable using preabsorbed Sprouty-1 antibody (C).

Fig. 2. Characterization of Sprouty-2 antibody by Western blot analysis of lung subcellular fractions. The fractions described in figure 1 were also used for characterization of Sprouty-2 antibody by Western blot analysis. A, B Blot treated with Sprouty2 antibody. C Blot treated with peptideabsorbed Sprouty-2 antibody. A specific band of approximately $34 \mathrm{kDa}$ was detected in the $13 \mathrm{~K}$ pellet fraction. This band was not detectable using preabsorbed Sprouty-2 antibody (C). No specific band was detected in the BAL or $13 \mathrm{~K}$ supernatant fractions (A).
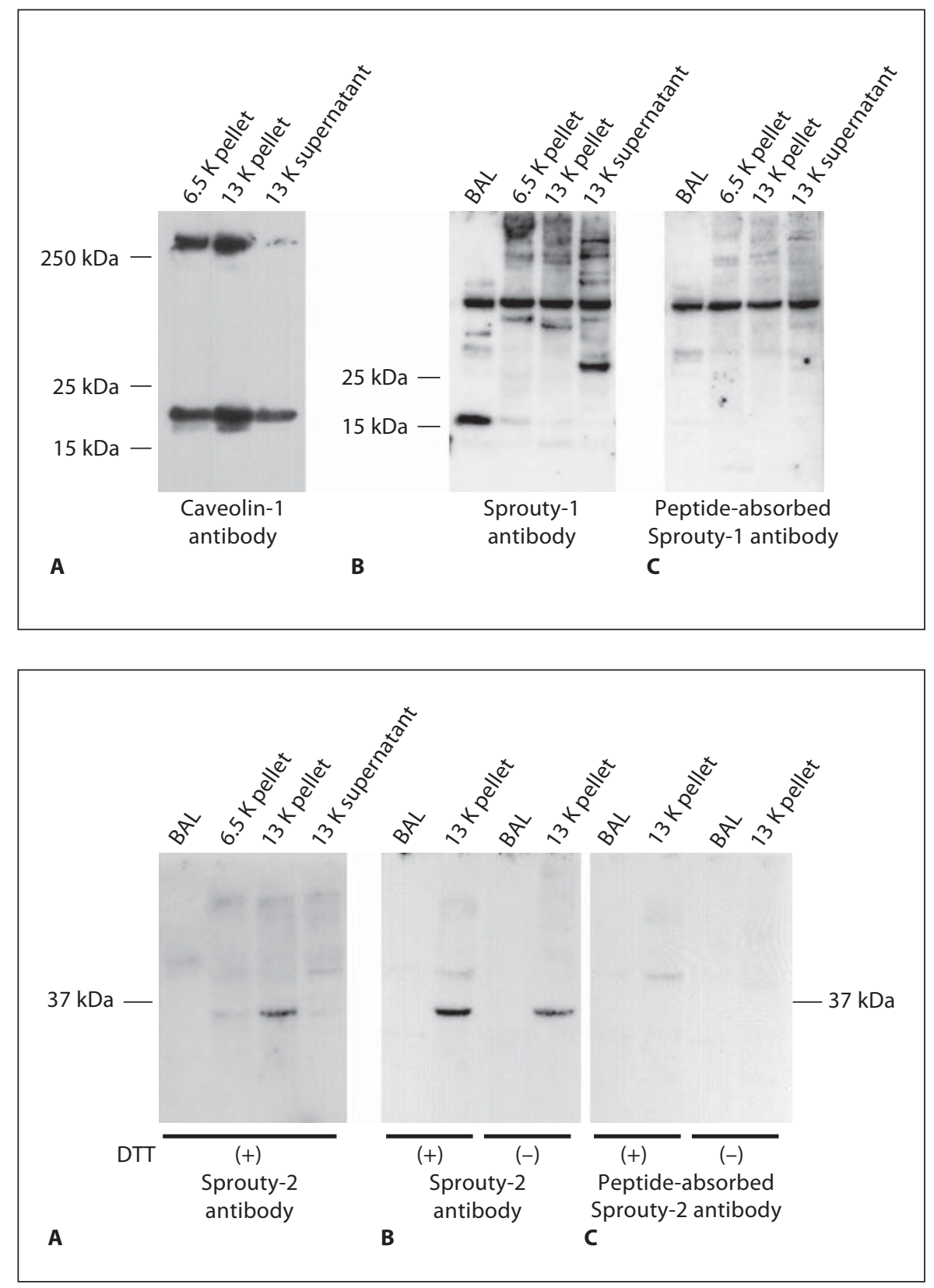

\section{Results}

Sprouty-1 and Sprouty-2 Proteins Have both Distinct and Overlapping Distribution Patterns in Lung

Subcellular Fractions

Three major fractions $(6.5 \mathrm{~K}$ pellet, $13 \mathrm{~K}$ pellet and 13 K supernatant) and BAL were examined by Western blot analysis using antibodies to caveolin-1 and Sprouty-1 (fig. 1) and Sprouty-2 (fig. 2). Caveolin-1 was included in the study because of its close intracellular association with
Sproutys [16]. The 6.5 and $13 \mathrm{~K}$ pellets and $13 \mathrm{~K}$ supernatant all showed the presence of caveolin-1, but the highest amount was found in the $13 \mathrm{~K}$ pellet fraction (fig. 1A). A band of about $30 \mathrm{kDa}$ was detected in the $13 \mathrm{~K}$ supernatant fraction with the Sprouty-1 antibody. The band was not detected in the 6.5 and $13 \mathrm{~K}$ pellet fractions or in BAL. However, an immunoreactive band of approximately 16 $\mathrm{kDa}$ was detected in BAL (fig. 1B). A $34-\mathrm{kDa}$ band was detected in the $13 \mathrm{~K}$ pellet fraction using Sprouty- 2 antibody (fig. 2A). No immunoreactive band was seen in the 


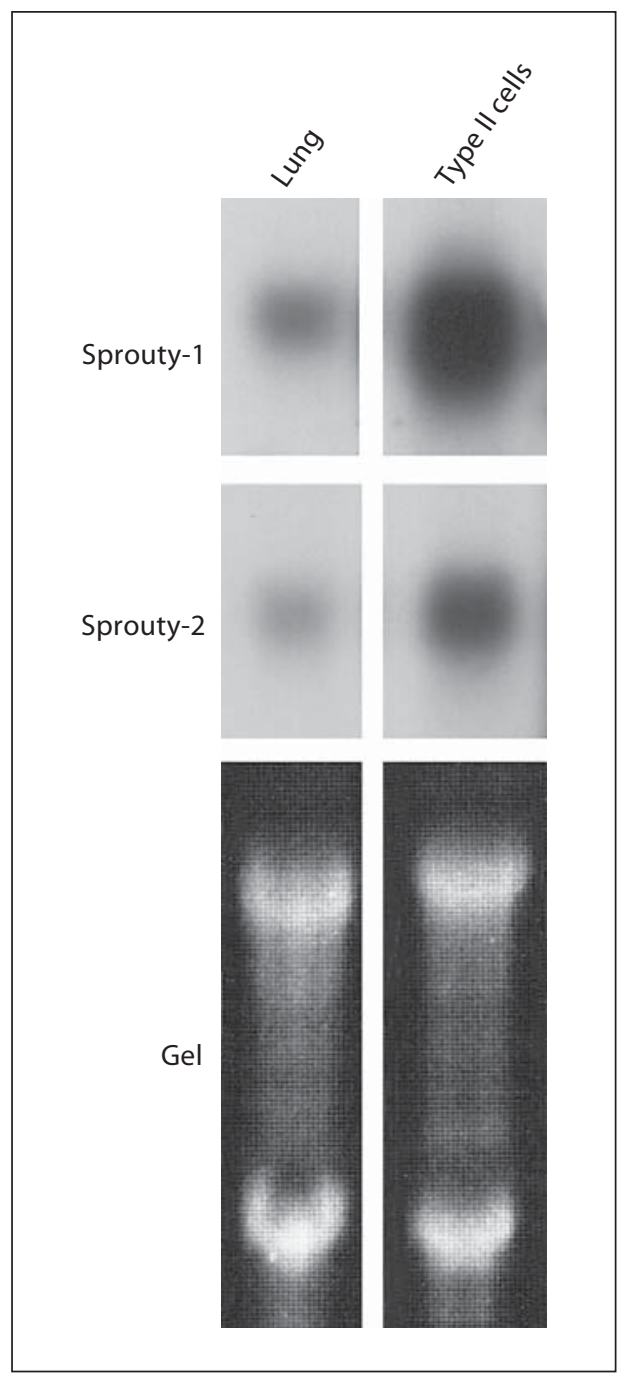

Fig. 3. Northern blot analysis of Sprouty-1 and Sprouty-2 mRNA expression in adult rat lung and isolated alveolar epithelial type II cells. High expression of mRNA of both Sproutys is seen in the lung and type II cells. The latter appear to be the major cell type expressing Sproutys in the adult rat lung.

$6.5 \mathrm{~K}$ pellet and $13 \mathrm{~K}$ supernatant fractions or in BAL using Sprouty-2 antibody (fig. 2A). These immunoreactive bands were not seen when the antibodies were preabsorbed with the Sprouty peptides (fig. 1C, 2C).

\section{Sprouty-1 and Sprouty-2 mRNA Expression in}

Isolated Type II Cells

To determine if Sproutys are actually expressed in rat lung, we investigated the expression of Sprouty-1 and Sprouty-2 mRNA in adult rat lung and isolated alveolar epithelial type II cells, which are one of the major com- ponents in lung epithelial cells (fig. 3). High expression of mRNA of both Sproutys was seen in the lung and type II cells. The latter appear to be the major cell type expressing Sproutys in adult rat lung.

\section{Localization of Sprouty-1 and -2 in Embryonic Rat Lung}

The distribution of Sprouty-1 and Sprouty-2 in embryonic day (E) 14, E16, E18 and adult rat lungs was determined by immunohistochemistry. In E14 lung, Sprouty-1 staining was seen throughout the airway epithelium, more predominantly at the basolateral surfaces of epithelial cells (fig. 4, arrows in B, D). Some of the mesenchymal cells adjacent to the epithelium and at the periphery were also stained (fig. 4, arrows in C, arrowheads in B, D). Sprouty-2 staining was seen in airway epithelium, more predominantly at the lumenal surfaces (fig. 5, arrows in C, D). Additionally, mesenchymal cells at the periphery were stained (fig. 5, arrowheads in B, D). In E16 lung, intense staining for both Sproutys was seen in the distal airway epithelium and adjoining peripheral mesenchymal cells (fig. 6). Uniform Sprouty-1 staining was seen at E16 in the distal airway epithelium (fig. 6, arrows in E), whereas Sprouty-2-stained cells were present at the leading edges of the distal epithelium (fig. 6, arrow in F). Sprouty-1 and Sprouty-2 staining was also detected in some of the epithelial cells of the proximal tubules (fig. 6, short arrows in C, D). In E18 lung, strong staining for Sprouty-1 and Sprouty-2 was restricted to the distal airway epithelium (fig. 7). The proximal airway epithelium was weakly stained for both proteins (fig. 7A, B). No staining was detected in peripheral mesenchyme adjoining the distal alveolar tip at this stage (fig. 7B-D).

\section{Comparison of mRNA Expression of Sprouty-1, \\ Sprouty-2 and Surfactant Protein C by \\ Dual-Probe in situ Hybridization in E18 Lungs}

To compare the cellular expression pattern of Sprouty-1, Sprouty-2 and surfactant protein (SP-C), which is usually expressed by type II cells, mRNA, we determined the expression of combinations of these mRNAs by dual-probe in situ hybridization in E18 lungs (fig. 8). Coexpression of Sprouty-1 or Sprouty-2 and SP-C mRNA was observed in epithelial cells of the distal alveolar buds (fig. 8, arrows in A, B). In addition, coexpression of Sprouty-1 and Sprouty-2 mRNA was observed in epithelial cells of the distal alveolar buds (fig. 8, arrows in C, D). Sprouty-1 mRNA was also expressed in the surrounding mesenchymal cells of the bronchioles (fig. 8, arrowheads 

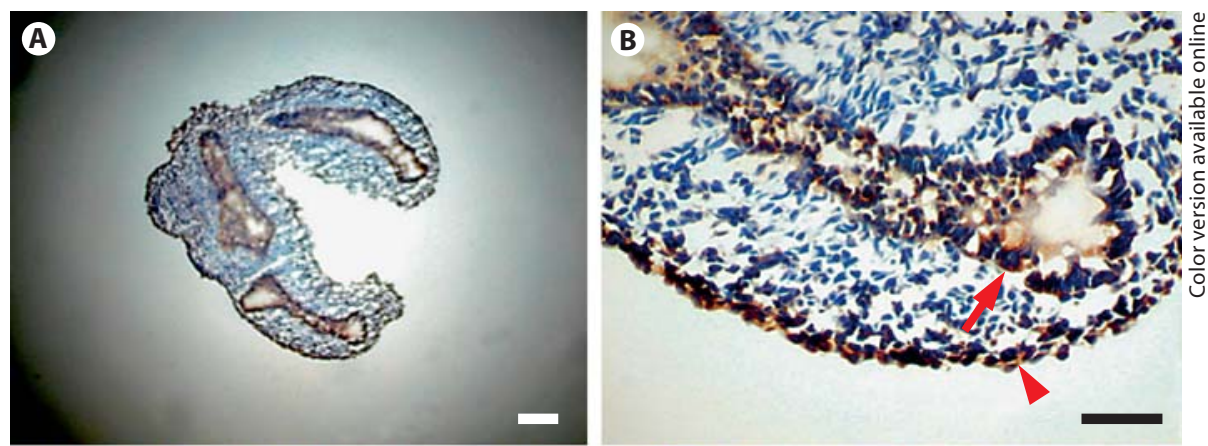

Fig. 4. Expression of Sprouty-1 in lung at the early pseudoglandular stage (E14). Sections from lungs of E14 rat embryos were immunostained with Sprouty-1 antibody (A). Staining for Sprouty-1 is seen at the basolateral surfaces of epithelial cells (arrows in B, D) and in mesenchymal cells adjacent to airway epithelium (arrows in $\mathbf{C}$ ) and at the periphery (arrowheads in B, D). Staining is especially strong in clusters of epithelial cells at the leading edges of branching airways. Bar $=100 \mu \mathrm{m}$.
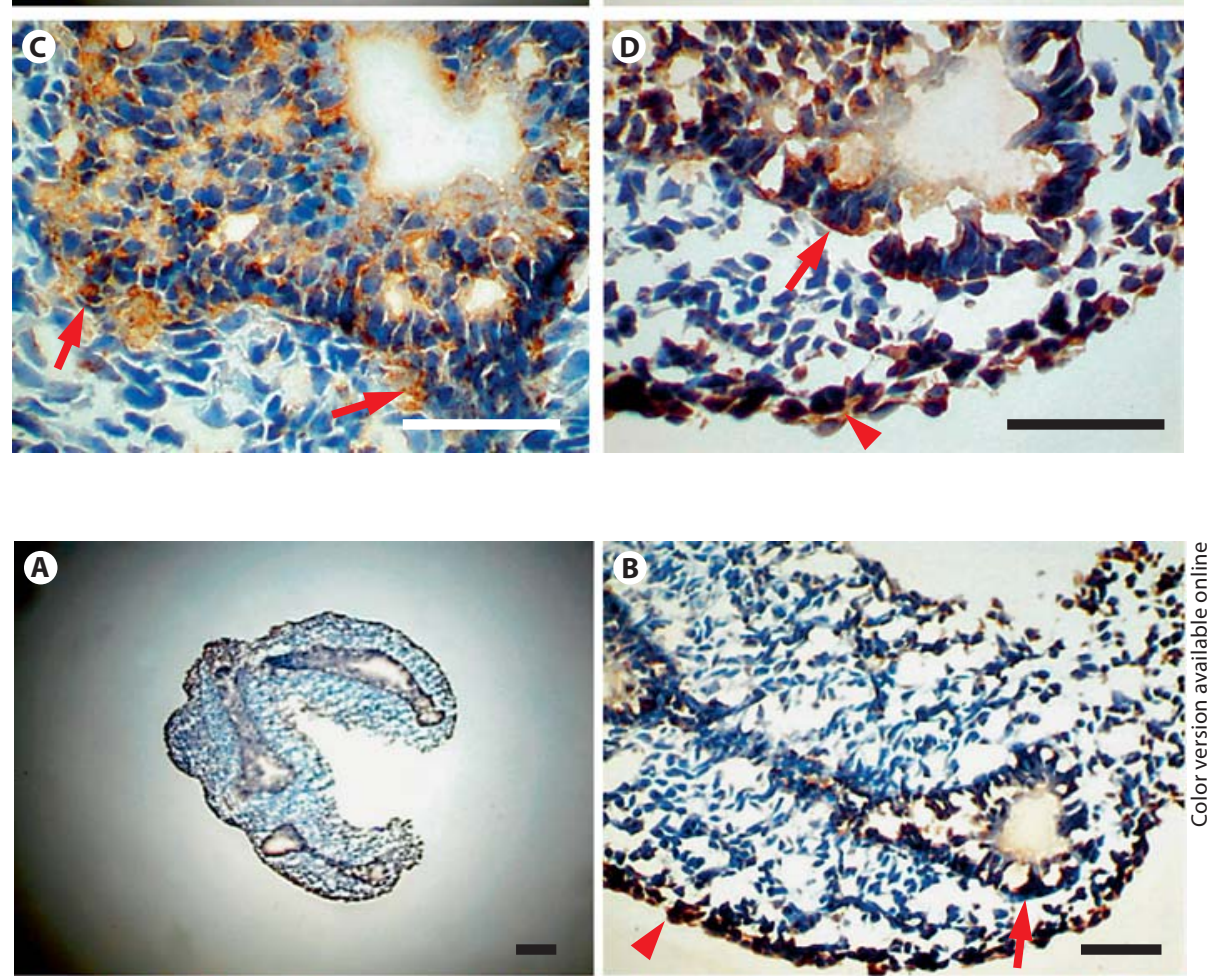

Fig. 5. Expression of Sprouty-2 in lung at the early pseudoglandular stage (E14). Sections from lungs of E14 rat embryos were immunostained with Sprouty-2 antibody (A). Staining for Sprouty-2 is seen at the lumenal surfaces of epithelial cells lining tubules (arrows in C, D) and in clusters of epithelial cells at the leading edges of branching tubules (arrow in B, arrowheads in C). Peripheral mesenchymal cells are also stained (arrowheads in B, D). $\operatorname{Bar}=100 \mu \mathrm{m}$.
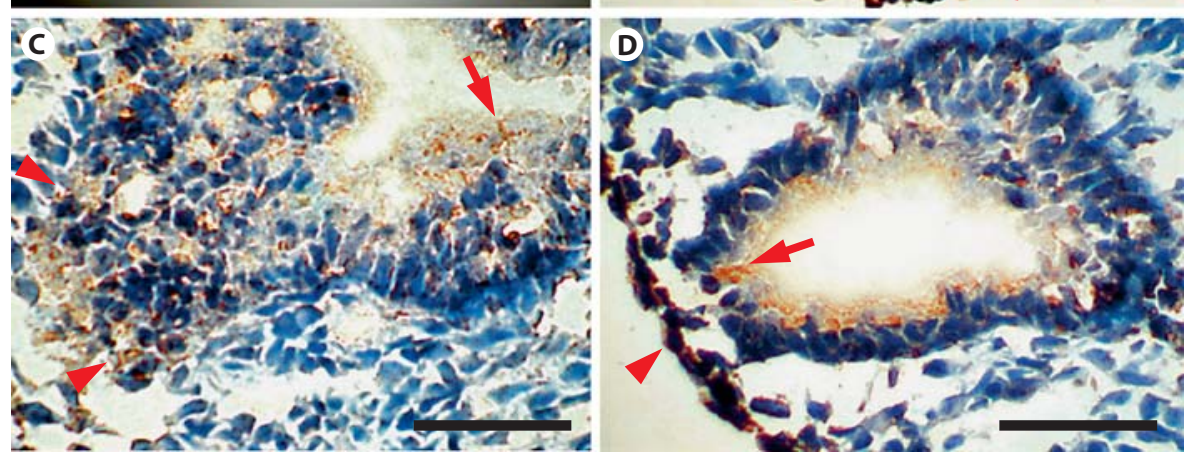

in A, C). From these findings, the overall distribution of Sprouty-1 and Sprouty-2 mRNA is overlapping in distal alveolar buds expressing SP-C mRNA and distinct in peribronchiolar mesencymal cells with regard to Sprouty-1 mRNA expression.
Localization of Sprouty-1 and Sprouty-2 in Adult Rat Lung

Expression of Sprouty-1 and Sprouty-2 was examined by immunohistochemistry in adult rat lungs and is shown in figures 9 and 10, respectively. Sprouty-1 expression was 
Fig. 6. Expression of Sprouty-1 and Sprouty-2 during the midpseudoglandular stage of lung development (E16). Sections from lungs of E16 rat embryos were immunostained with Sprouty-1 antibody (A, C, E) or Sprouty-2 antibody (B, D, F). Staining for Sprouty-1 and Sprouty-2 is seen in the epithelium of distal tubules (long arrows in $\mathbf{C}-\mathbf{F}$ ) as well as in the peripheral mesenchymal cells (arrowheads in E, F). While all epithelial cells of distal tubules are uniformly stained for Sprouty-1 (arrows in E), staining for Sprouty-2 is limited to the epithelial cells closer to the periphery of the lung (arrow in F). Low levels of Sprouty-1 and Sprouty-2 were detected in mesenchyme (arrowheads in C, D) and proximal airway epithelium (short arrows in C, D). Bar $=100 \mu \mathrm{m}$.
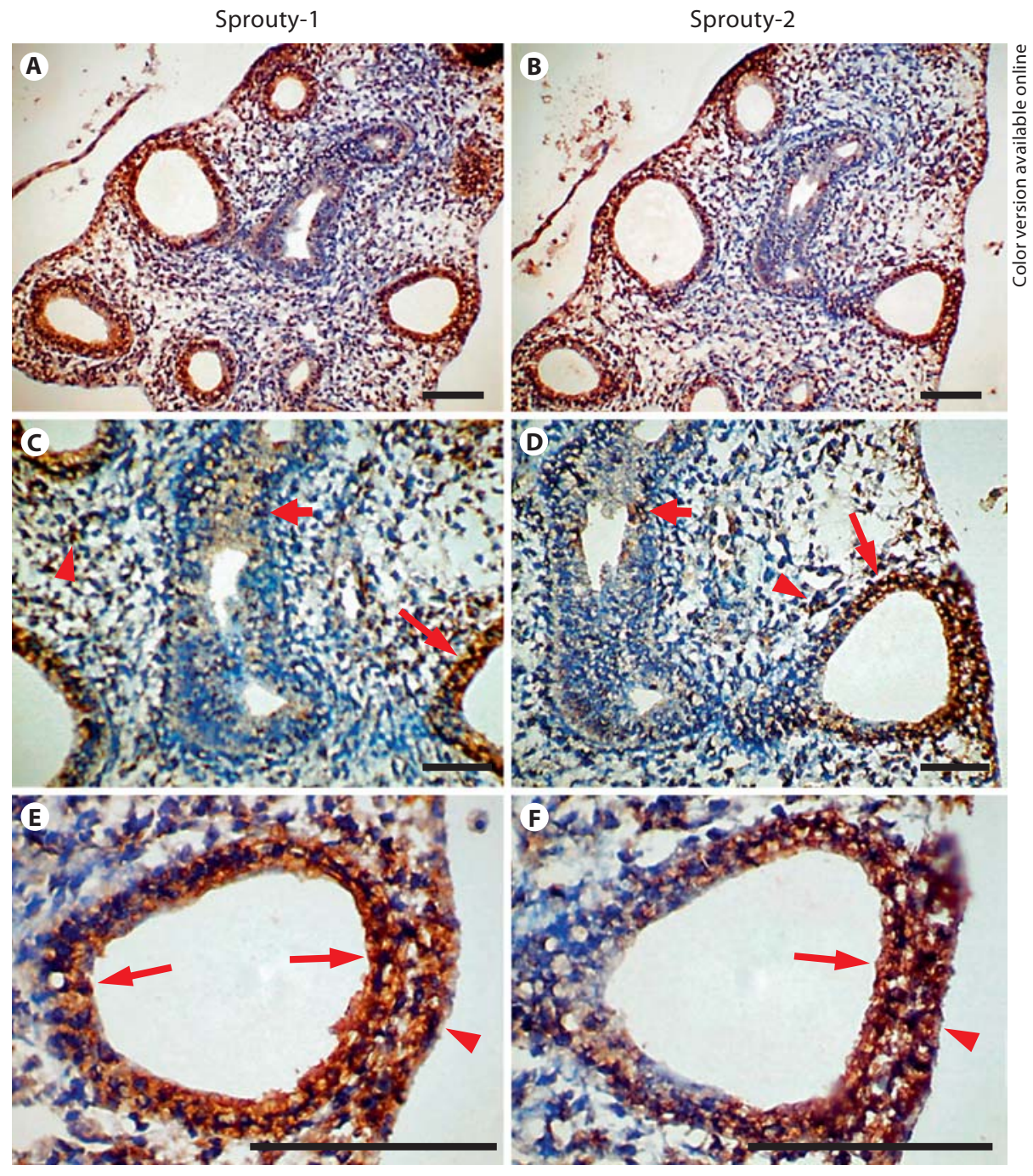

strong in the bronchiolar epithelium (fig. 9B, C). In the alveolar region, epithelial type I and type II cells were frequently stained (fig. 9D). Intense staining for Sprouty-2 was also seen in the bronchiolar epithelial cells (fig. 10B, C). In these cells, staining was often seen in membranes surrounding cytoplasmic vacuoles/vesicles (fig. 10, arrows in C, inset). Sprouty-2 expression was also seen in alveolar epithelial type II cells (fig. 10, arrows in D). No staining was detected when Sprouty-1 and Sprouty-2 peptide-absorbed antibodies were used for staining (fig. 9A, 10A). Overall, Sprouty-1 and Sprouty-2 protein distribution was similar to our previously reported mRNA expression [11].

\section{Distribution of Sprouty-1 and Sprouty-2 mRNA}

Determined by in situ Hybridization

To verify the cellular expression of Sprouty-1 and Sprouty-2 by immunohistochemistry, we performed in situ hybridization to identify the cellular distribution of mRNA expression of both Sproutys in E18 lungs by comparing their expression with Clara cell $10-\mathrm{kDa}$ protein (CC10), which is usually expressed and secreted by Clara cells, and SP-C mRNA expression (fig. 11). Sprouty- 1 and, to a lesser extent, Sprouty-2 mRNAs were expressed in the bronchiolar epithelial cells (fig. 11, arrows in C, D). In the alveolar region, mRNA expression of both Sproutys was seen in type II cells, which were cuboidal and usually located at the corner of the alveoli (fig. 11, arrows in E, F). In addition, expression of Sprouty- 1 mRNA was also seen in type I cells, which were flattened and usually located between the corners of the alveoli (fig. 11, arrowheads in E). Overall, these results were similar to the Sprouty- 1 and Sprouty-2 protein distribution and to our previous findings for mRNA expression in the developing and adult rat lung [11]. 
Fig. 7. Sprouty-1 and Sprouty-2 expression in E18 lung. Sections of E18 lungs were immunostained using Sprouty-1 (A, C) and Sprouty-2 (B, D) antibodies. Strong staining for both Sproutys is seen in the distal epithelium (arrows in A-D). Expression of these proteins is weak in the proximal epithelium (arrowheads in A, B). Peripheral mesenchymal staining is barely detected at this stage of lung development (arrowheads in C, D). Bi = Bronchiole; $\mathrm{Al}=$ alveolus. $\operatorname{Bar}=100 \mu \mathrm{m}$.

Fig. 8. Dual-probe in situ hybridization to monitor the expression of SP-C, Sprouty-1 and Sprouty-2 mRNA in E18 lung. A Overlapping expression of Sprouty-1 (red; colors only in online version) and SP-C (brown) mRNA was observed in epithelial cells of the distal alveolar buds (arrows), whereas only Sprouty-1 mRNA expression was observed in the peribronchiolar mesenchymal cells (arrowheads). B Overlapping expression of Sprouty-2 (red) and SP-C (brown) mRNA is also seen in the epithelial cells of the distal alveolar buds (arrows). Sprouty-2 mRNA expression is not seen in peribronchiolar mesenchymal cells (arrowhead). C, D Overall distribution of Sprouty-1 (brown) and Sprouty-2 (red) mRNA is overlapping in distal alveolar buds (arrows in C, D) and distinct in peribronchiolar mesenchymal cells with regard to Sprouty-1 mRNA expression (arrowheads in $\mathbf{C}$ ). $\mathrm{Bi}=$ Bronchiole; $\mathrm{Al}=$ alveolus. $\mathrm{Bar}=100 \mu \mathrm{m}$.
Sprouty-1
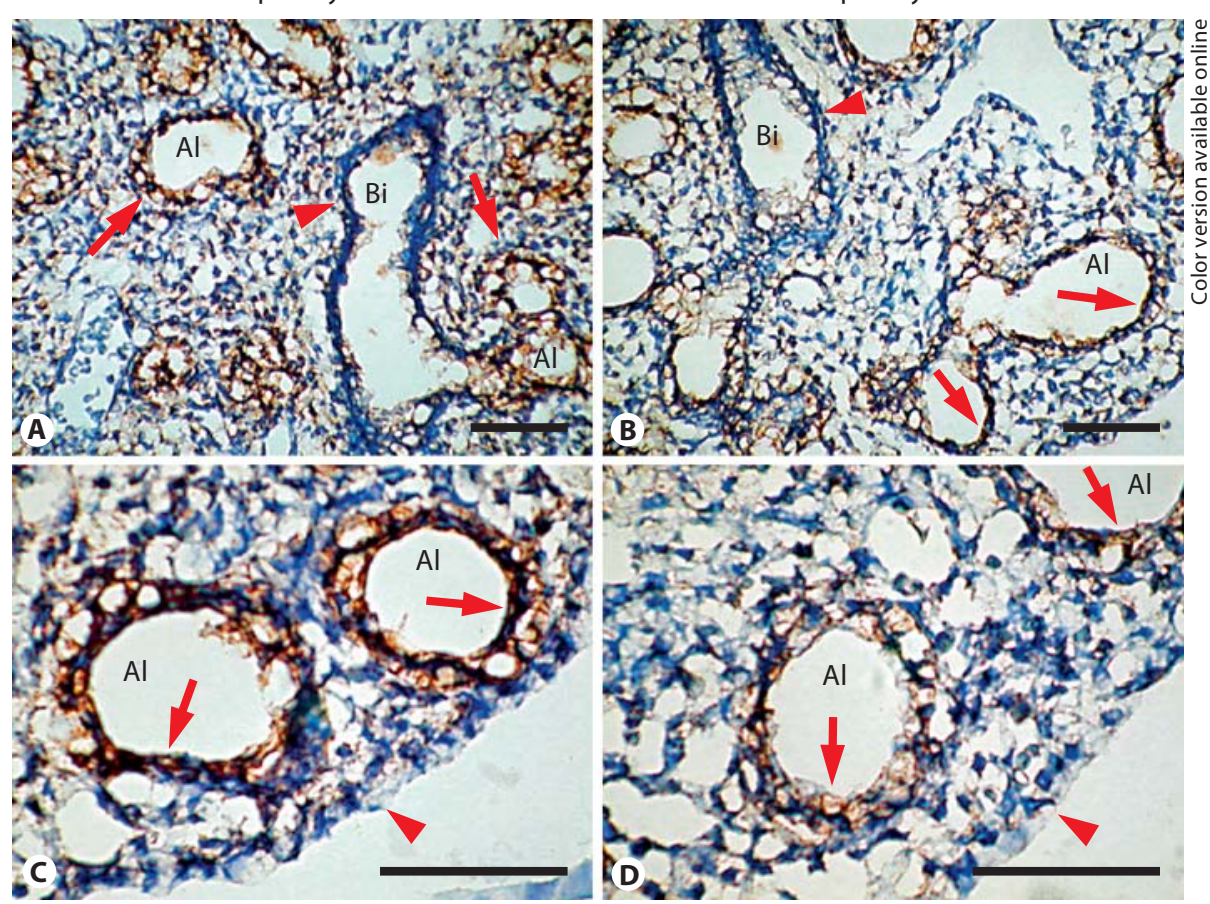
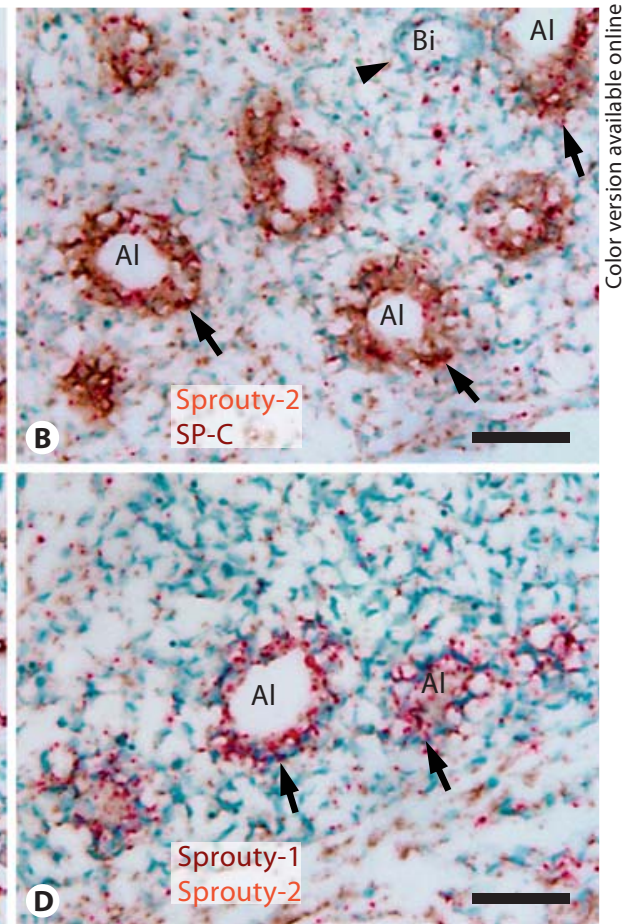
Fig. 9. Sprouty-1 expression in adult rat lung. Sections of adult rat lung were immunostained using Sprouty-1 antibody. A Treatment with peptide-absorbed Sprouty-1 antibody. B, C Bronchiolar epithelium at different magnifications. D Alveolar epithelium. Bronchial epithelium shows strong staining (arrows in B, C). In the alveolar region, type II (arrows in D) and type I epithelial cells (arrowheads in D) are frequently stained. There is no staining in the section treated with preabsorbed antibody (A). Bi = Bronchiole; $\mathrm{Al}=$ alveolus; $\mathrm{V}=$ vessel. $\mathrm{Bar}=100 \mu \mathrm{m}$.
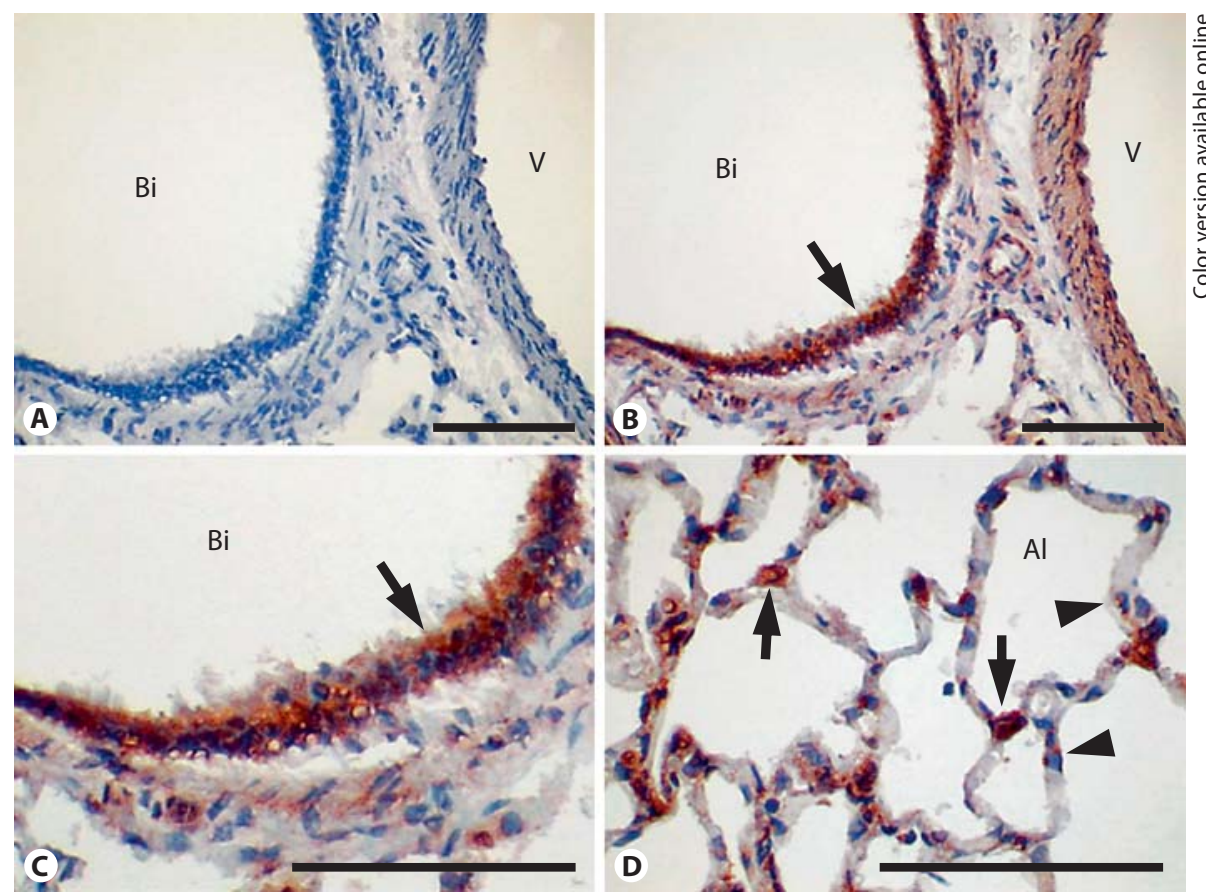

Fig. 10. Sprouty-2 expression in adult rat lung. Sections of adult rat lung were immunostained using Sprouty-2 antibody. A Treatment with peptide-absorbed Sprouty-2 antibody. B, C Bronchiolar epithelium at different magnifications. D Alveolar epithelium. Intense staining is seen in bronchial epithelial cells. In these cells, the membranes surrounding the intracellular vacuoles/vesicles are also stained (arrows in B, C and inset). In the alveolar region, type II epithelial cells are frequently stained (arrows in D). No staining is seen in sections treated with preabsorbed antibody $(\mathbf{A})$. $\mathrm{Bi}=$ Bronchiole; $\mathrm{Al}=$ Alveolus. $\operatorname{Bar}=100 \mu \mathrm{m}$.

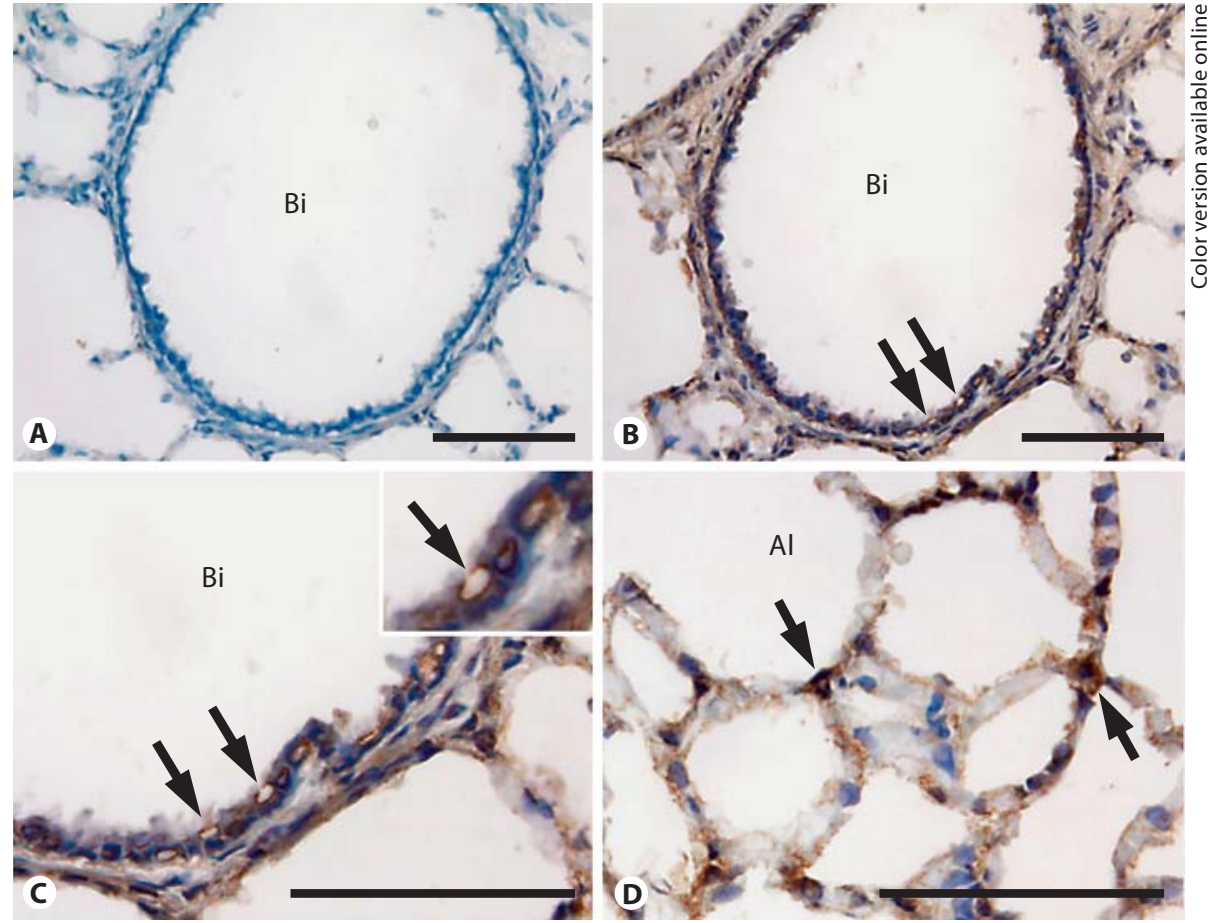


Fig. 11. In situ hybridization to compare the expression of Sprouty-1, Sprouty-2, CC10 and SP-C mRNA in the adult rat lung. A Bronchiolar epithelial cells show high CC10 mRNA expression (arrow). CC10 mRNA expression is also seen in a smaller number of alveolar epithelial type II cells (arrowhead). B In contrast, a distinct distribution of SP-C mRNA in alveolar epithelial type II cells was observed (arrow), with expression in a smaller number of bronchiolar epithelial cells (arrowhead). C, D Sprouty-1 and, to a lesser extent, Sprouty-2 mRNA are expressed in the bronchiolar epithelial cells (arrows). E, F In the alveolar region, expression of both Sproutys is seen in type II cells (arrows). Sprouty-1 mRNA is also expressed in type I cells (arrowheads in E). Bi = Bronchiole; $\mathrm{Al}=$ alveolus. $\mathrm{Bar}=100 \mu \mathrm{m}$.

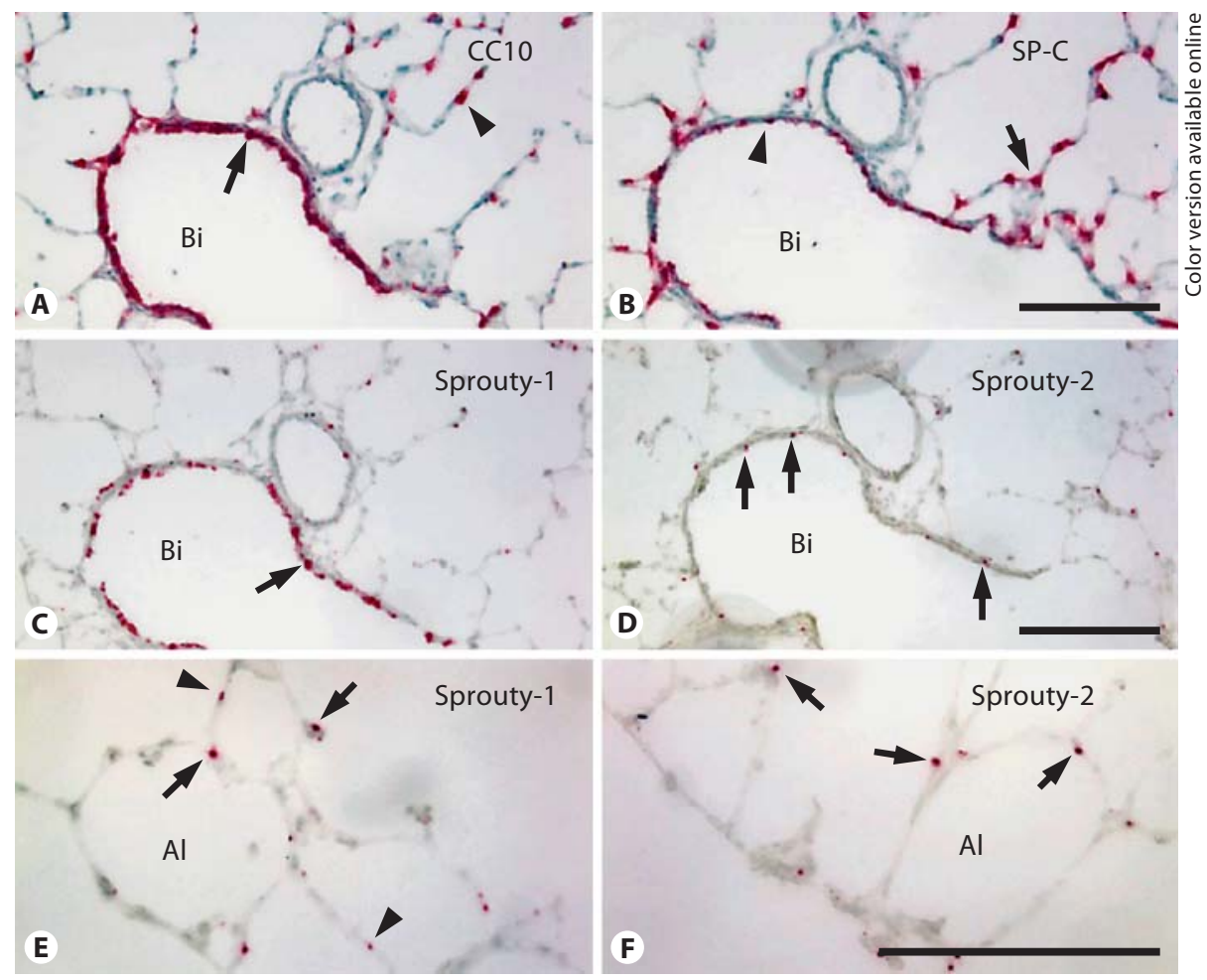

\section{Discussion}

Sprouty has been shown to inhibit signaling by several tyrosine kinase receptors [3-5, 7]. In Drosophila, Sprouty acts as an antagonist of fibroblast growth factor (FGF) signaling and its expression is induced by the same signaling pathway that it inhibits [4]. Drosophila Sprouty is known to regulate tracheal branching by limiting the action of Branchless, an FGF that acts as an inducer of branching [4]. The present study demonstrates that Sprouty-1 and Sprouty-2 proteins are expressed in early embryonic lung both in the epithelial cells of distal tubules and in peripheral mesenchymal cells. Peripheral mesenchymal cells are also known to express FGF-10, which induces airway branching by its action as a chemoattractant on advancing tubular epithelium $[6,11,17,18]$. FGF-10 is required for lung development; FGF-10 knockout animals lack lungs $[19,20]$. It is not known whether Sprouty expression in distal epithelial and mesenchymal cells is in response to FGF-10 or whether other growth factor(s) released in the vicinity induce their expression. A potential candidate is FGF-9, which is expressed by mesothelial cells and also by distal epithelium from the midpseudoglandular stage in embryonic mouse lung and up-regulates Fgf-10 ex- pression in the mesenchyme [21]. In addition, it was also recently reported that FGF-9 signaling stimulates Spred and Sprouty expression in embryonic mouse pancreas mesenchyme [22]. These findings indicate that FGF-9 may also regulate Sprouty expression in neighboring mesenchymal and epithelial cells in embryonic rat lung development. Overall, our results point to close proximity of Sprouty-1 and Sprouty-2 expression to the expression domains of FGF, similar to the findings reported by another group in the development of many organs [9]. In E14 and E16 lungs, Sprouty-1 and Sprouty-2 were expressed in epithelial and mesenchymal cells, whereas in E18 lungs at the completion of branching morphogenesis, the expression of these proteins was not detected in peripheral mesenchymal cells and was restricted to epithelial cells. These findings suggest that normal branching morphogenesis may require mesenchymal Sprouty expression.

In adult rat lung, Sprouty-1 and Sprouty- 2 mRNAs and proteins are present in alveolar epithelial type II cells. While current evidence suggests that Sprouty-2 is a key regulator of branching morphogenesis [10], its function as well as that of other Sprouty homologs during branching morphogenesis and later stages of lung development and in the adult rat lung remain to be investigated. There 
is some evidence suggesting that Sprouty may play a role in cell differentiation contrary to its established role as a general inhibitor of receptor tyrosine kinase signaling. Wong et al. [23] demonstrated that both Sprouty-1 and Sprouty-2 abrogate epidermal growth factor receptor ubiquitylation and endocytosis, enhance epidermal growth factor-induced ERK signaling and induce differentiation of PC12 cells. It has also been reported that Sprouty-2 regulates oncogenic K-ras in lung development and in the context of K-ras ${ }^{\mathrm{G} 12 \mathrm{D}}$-mediated lung tumorigenesis by limiting tumor number and overall tumor burden [24].

The presence of a $16-\mathrm{kDa}$ polypeptide in BAL is a new and potentially important finding. The polypeptide may have resulted from proteolytic processing of nascent Sprouty-1 $(30 \mathrm{kDa})$. There are two potential proteolytic cleavage sites consisting of RXXR sequences. These sequences are present in Sprouty-1 protein at amino acids 143-146 and 146-149. Cleavage at these sites would yield a fragment of approximately $16 \mathrm{kDa}$, recognizable by Western blot analysis using the antibody to a Sprouty-1 peptide (amino acids 20-33). It is not yet clear whether the protein processing occurs intracellularly with the se- cretion of the fragment or extracellularly after secretion by the cell. Whether Sprouty proteins are actively secreted is controversial. The initial studies on Drosophila Sprouty suggested it has a cell nonautonomous action as an extracellular antagonist of FGF signaling $[4,5]$. Sprouty protein was found to be localized in a punctate pattern resembling intracellular endocytic uptake vesicles or secretory vesicles in developing eye discs [5]. In contrast, there is evidence that Sprouty homologs are intracellular antagonists of receptor tyrosine kinase pathways and are localized at the inner surface of the plasma membrane $[3,16,25]$. Future studies would determine the nature and site of Sprouty-1 protein processing in the lung and the function, if any, of the $16-\mathrm{kDa}$ Sprouty- 1 fragment.

\section{Acknowledgements}

This work was supported by a grant from National Institutes of Health (HL 62397). We thank Dr. X. Guo for nucleotide sequencing of rat Sprouty-1 and Sprouty-2 cDNAs.

\section{References}

1 Metzger RJ, Klein OD, Martin GR, Krasnow MA: The branching programme of mouse lung development. Nature 2008;453:745750 .

2 Morrisey EE, Hogan BL: Preparing for the first breath: genetic and cellular mechanisms in lung development. Dev Cell 2010; 18:8-23.

3 Casci T, Vinos J, Freeman M: Sprouty, an intracellular inhibitor of ras signaling. Cell 1999;96:655-665

4 Hacohen N, Kramer S, Sutherland D, Hiromi Y, Krasnow MA: Sprouty encodes a novel antagonist of FGF signaling that patterns apical branching of the Drosophila airways. Cell 1998;92:253-263.

5 Kramer S, Okabe M, Hacohen N, Krasnow MA, Hiromi Y: Sprouty: a common antagonist of FGF and EGF signaling pathways in Drosophila. Development 1999;126:25152525.

-6 Mailleux AA, Tefft D, Ndiaye D, Itoh N, Thiery JP, Warburton D, Bellusci S: Evidence that sprouty2 functions as an inhibitor of mouse embryonic lung growth and morphogenesis. Mech Dev 2001;102:81-94.

7 Reich A, Sapir A, Shilo B: Sprouty is a general inhibitor of receptor tyrosine kinase signaling. Development 1999;126:4139-4147.
8 de Maximy AA, Nakatake Y, Moncada S, Itoh N, Thiery JP, Bellusci S: Cloning and expression pattern of a mouse homologue of Drosophila sprouty in the mouse embryo. Mech Dev 1999;81:213-216.

-9 Minowada G, Jarvis LA, Chi CL, Neubuser A, Sun X, Hacohen N, Krasnow MA, Martin GR: Vertebrate Sprouty genes are induced by FGF signaling and can cause chondrodysplasia when overexpressed. Development 1999; 126:4465-4475.

10 Tefft JD, Lee M, Smith S, Leinwand M, Zhao J, Bringas P Jr, Crowe DL, Warburton D: Conserved function of mSpry-2, a murine homolog of Drosophila sprouty, which negatively modulates respiratory organogenesis. Curr Biol 1999;9:219-222.

11 Hashimoto S, Nakano H, Singh G, Katyal S: Expression of spred and sprouty in developing rat lung. Mech Dev 2002;119(suppl 1):S303-S309.

12 Zhang S, Lin Y, Itaranta P, Yagi A, Vainio S: Expression of sprouty genes 1, 2 and 4 during mouse organogenesis. Mech Dev 2001;109: $367-370$
13 Oshika E, Liu S, Ung LP, Singh G, Shinozuka H, Michalopoulos GK, Katyal SL: Glucocorticoid-induced effects on pattern formation and epithelial cell differentiation in early embryonic rat lungs. Pediatr Res 1998;43: 305-314.

14 Shiratori M, Michalopoulos G, Shinozuka H, Singh G, Ogasawara H, Katyal SL: Hepatocyte growth factor stimulates DNA synthesis in alveolar epithelial type II cells in vitro. Am J Respir Cell Mol Biol 1995;12: 171-180.

15 Shiratori M, Oshika E, Ung LP, Singh G, Shinozuka H, Warburton D, Michalopoulos G, Katyal SL: Keratinocyte growth factor and embryonic rat lung morphogenesis. Am J Respir Cell Mol Biol 1996;15:328-338.

16 Impagnatiello MA, Weitzer S, Gannon G, Compagni A, Cotten M, Christofori G: Mammalian sprouty-1 and -2 are membrane-anchored phosphoprotein inhibitors of growth factor signaling in endothelial cells. J Cell Biol 2001;152:1087-1098.

17 Bellusci S, Grindley J, Emoto H, Itoh N, Hogan BL: Fibroblast growth factor 10 (FGF10) and branching morphogenesis in the embryonic mouse lung. Development 1997;124: 4867-4878. 
-18 Park WY, Miranda B, Lebeche D, Hashimoto G, Cardoso WV: FGF-10 is a chemotactic factor for distal epithelial buds during lung development. Dev Biol 1998;201:125-134.

-19 Min H, Danilenko DM, Scully SA, Bolon B, Ring BD, Tarpley JE, DeRose M, Simonet WS: FGF-10 is required for both limb and lung development and exhibits striking functional similarity to Drosophila branchless. Genes Dev 1998;12:3156-3161.

20 Sekine K, Ohuchi H, Fujiwara M, Yamasaki M, Yoshizawa T, Sato T, Yagishita N, Matsui D, Koga Y, Itoh N, Kato S: FGF10 is essential for limb and lung formation. Nat Genet 1999; 21:138-141.
21 del Moral PM, De Langhe SP, Sala FG, Veltmaat JM, Tefft D, Wang K, Warburton D, Bellusci S: Differential role of FGF9 on epithelium and mesenchyme in mouse embryonic lung. Dev Biol 2006;293:77-89.

22 Sylvestersen KB, Herrera PL, Serup P, Rescan C: Fgf9 signalling stimulates Spred and Sprouty expression in embryonic mouse pancreas mesenchyme. Gene Expr Patterns 2011;11:105-111.

23 Wong ES, Fong CW, Lim J, Yusoff P, Low BC, Langdon WY, Guy GR: Sprouty2 attenuates epidermal growth factor receptor ubiquitylation and endocytosis, and consequently enhances Ras/ERK signalling. EMBO J 2002; 21:4796-4808
24 Shaw AT, Meissner A, Dowdle JA, Crowley D, Magendantz M, Ouyang C, Parisi T, Rajagopal J, Blank LJ, Bronson RT, Stone JR, Tuveson DA, Jaenisch R, Jacks T: Sprouty-2 regulates oncogenic K-ras in lung development and tumorigenesis. Genes Dev 2007;21:694707.

25 Lim J, Wong ES, Ong SH, Yusoff P, Low BC, Guy GR: Sprouty proteins are targeted to membrane ruffles upon growth factor receptor tyrosine kinase activation. Identification of a novel translocation domain. J Biol Chem 2000;275:32837-32845. 